\title{
Electrical conductance between conductors with dissimilar temperature-dependent material properties
}

\author{
Yong Hoon Jang, J R Barber and S Jack Hu \\ Department of Mechanical Engineering and Applied Mechanics, University of \\ Michigan, Ann Arbor, MI 48109-2125, USA
}

Received 18 May 1998

\begin{abstract}
The method of Greenwood and Williamson is extended to give a general solution for the coupled nonlinear problem of steady-state electrical and thermal conduction across an interface between two conductors of dissimilar materials, for both of which the electrical resistivity and thermal conductivity are functions of temperature. The method presented is sufficiently general to cover all combinations of conductor geometry, material properties and boundary values provided that (i) the current enters and leaves the conductor through two equipotential isothermal surfaces, (ii) the remaining boundaries of the conductor are thermally and electrically insulated and (iii) the interface(s) between different materials would be equipotential surfaces in the corresponding linear problem. Under these restrictions, the problem can be decomposed into the solution of a pair of nonlinear algebraic equations involving the boundary values and the material properties, followed by a linear mapping of the resulting one-dimensional solution into the actual conductor geometry. Examples are given involving single and multiple contact areas between dissimilar half spaces.
\end{abstract}

\section{Introduction}

Electrical conduction across an interface between two conductors is a problem of considerable technical importance, with applications in resistance welding (Thornton et al 1996), electrical connectors (Bryant 1994) and electrical machinery (Yune and Bryant 1988). Conditions at the interface can be extremely complex because of the roughness of the surfaces and the presence of insulating surface films and layers (Holm 1967, Runde 1987). In addition, electrical resistivity of the material leads to the generation of heat, causing high local temperatures at or near areas of actual electrical contact. Furthermore, the electrical resistivity generally increases quite significantly with temperature, causing nonlinear coupling between the electrical and thermal problems and in some cases leading to localization instabilities.

Electrical flow problems in which the resistivities vary with temperature are in general intractable. However, Kohlrausch (1900) has shown that the equipotential surfaces and the isothermal surfaces in a conductor will coincide in the steady state, leading to a one-to-one relation between temperature and potential, as long as the current enters and leaves the conductor through two equipotential isothermal surfaces and the remaining boundaries of the conductor are thermally and electrically insulated. Greenwood and Williamson (1958) applied Kohlrausch's results and additional results due to Diesselhorst (1900) to the problem in which an electric current is conducted through a single circular contact area between two large conductors of similar materials with temperature-dependent properties. However, they considered only the case where the temperatures of the two bodies distant from the interface are equal and hence where the maximum temperature, by symmetry, occurs at the interfacial plane. The more general case where the boundary temperatures are dissimilar was recently treated by Fournet (1997), including the situation in which the temperature varies monotonically through the bodies, so that the maximum temperature isotherm does not occur within the bodies.

In many practical applications, the materials of the two conductors will be different-for example carbon brushes contacting a copper commutator or copper electrodes contacting a steel workpiece in welding. Timisit (1988) has given an approximate solution for a problem of this class, but it is based on the restrictive assumption that the maximum temperature will coincide with the surface whose potential is midway between those of the equipotential boundaries. However, this assumption is not even a good approximation for the general case where the conductors 
may have arbitrary shape and the temperatures at the boundaries may be unequal.

In the present paper, we shall extend the methods of Greenwood and Williamson to the problem of steadystate electrical and thermal conduction across an interface between two conductors of dissimilar materials, for both of which the electrical resistivity and thermal conductivity can be fairly general functions of temperature. We shall demonstrate that the same methods can be rigorously applied provided that the interface is an equipotential surface in the corresponding linear problem. We also show that the problem can be decomposed into the solution of a pair of nonlinear algebraic equations involving the boundary values and the material properties, followed by a linear mapping of the resulting one-dimensional solution into the actual conductor geometry. The method presented is sufficiently general to cover all combinations of conductor geometry, material properties and boundary values. Finally, some examples are given concerning single and multiple contact areas between dissimilar half spaces.

\section{General considerations}

Ohm's law of electrical conduction requires that the current density

$$
\boldsymbol{J}=\frac{1}{\rho} \nabla \phi
$$

where $\phi$ is the electrical potential and $\rho$ is the electrical resistivity. Kirchoff's law then requires that

$$
\operatorname{div} \boldsymbol{J}=\operatorname{div}\left(\frac{1}{\rho} \nabla \phi\right)=0 .
$$

The resistive losses in the material lead to the generation of heat $q=|\boldsymbol{J}|^{2} \rho$ per unit volume in the medium and the steady-state heat conduction equation then requires that the heat generated per unit volume

$$
Q=-\operatorname{div} \boldsymbol{q}=-\operatorname{div}(\lambda \nabla \theta)=\frac{1}{\rho}|\nabla \phi|^{2}
$$

where $\boldsymbol{q}$ is the heat flux vector, $\theta$ is temperature and $\lambda$ is the thermal conductivity. Both $\rho$ and $\lambda$ are assumed to be functions of temperature $\theta$.

\subsection{The relation between temperature and potential}

Consider a body, $\Omega$, of a single material, such that current enters and leaves through two equipotential and isothermal surfaces $S_{1}, \quad S_{2}$ at $\left(\phi_{1}, \theta_{1}\right)$ and $\left(\phi_{2}, \theta_{2}\right)$ respectively, the remaining surfaces being thermally and electrically insulated. Greenwood and Williamson (1958) have shown that in this case there is a unique relation between temperature and potential throughout $\Omega$ which can be written in the simple form

$$
\phi= \pm \sqrt{2 \int_{\theta}^{\theta_{m}} \lambda \rho \mathrm{d} \theta}
$$

where $\theta_{m}$ is the maximum temperature and we have adopted the convention that the zero of potential is taken at the point where $\theta=\theta_{m}$. With this convention, the isotherm $\theta=\theta_{m}$ separates $\Omega$ into two parts, in one of which the positive sign is taken in equation (4), the negative sign being taken in the other part.

Circumstances can arise in which the temperature varies monotonically through the body and hence in which the maximum of temperature does not occur within the body. Problems of this class can be treated by considering $\Omega$ to be part of some larger fictitious body in which $\theta_{m}$ occurs. To determine which of these cases obtains, we first define the quantity

$$
X=\int_{\theta_{1}}^{\theta_{2}} \lambda \rho \mathrm{d} \theta=\phi_{1}^{2}-\phi_{2}^{2}=U\left(\phi_{1}+\phi_{2}\right)
$$

where $U=\left(\phi_{1}-\phi_{2}\right)$ is the potential difference. We can then write

$$
\begin{aligned}
& 2 \phi_{1} \equiv\left(\phi_{1}+\phi_{2}\right)+\left(\phi_{1}-\phi_{2}\right)=\frac{X}{U}+U \\
& 2 \phi_{2} \equiv\left(\phi_{1}+\phi_{2}\right)-\left(\phi_{1}-\phi_{2}\right)=\frac{X}{U}-U
\end{aligned}
$$

and multiplying these expressions, we have

$$
4 \phi_{1} \phi_{2}=\frac{X^{2}}{U^{2}}-U^{2}
$$

It follows that if $U^{4}>X^{2}, \phi_{1} \phi_{2}<0$ and hence that the maximum temperature isotherm lies within $\Omega$, whereas if $U^{4}<X^{2}, \phi_{1} \phi_{2}>0$ and the temperature varies monotonically through $\Omega$.

In a practical problem the temperatures $\theta_{1}, \theta_{2}$ and the potential difference $U$ will be known, We can choose to label the boundaries $S_{1}, S_{2}$ such that $\theta_{2}>\theta_{1}$ without loss of generality. It then follows from equation (4) that $\left|\phi_{1}\right|>\left|\phi_{2}\right|$ and it can be shown that the appropriate signs to take in equation (4) are then

$$
\begin{gathered}
\phi_{1}=\operatorname{sgn}(U) \sqrt{2 \int_{\theta_{1}}^{\theta_{m}} \lambda \rho \mathrm{d} \theta} \\
\phi_{2}=\operatorname{sgn}(U) \operatorname{sgn}\left(X^{2}-U^{4}\right) \sqrt{2 \int_{\theta_{2}}^{\theta_{m}} \lambda \rho \mathrm{d} \theta}
\end{gathered}
$$

where $\operatorname{sgn}(x)$ is the sigmum function which equals 1 for $x>0$ and -1 for $x<0$.

Substituting these results into the equation $U=\phi_{1}-\phi_{2}$ and simplifying, we obtain the nonlinear equation

$$
|U|=\sqrt{2 \int_{\theta_{1}}^{\theta_{m}} \lambda \rho \mathrm{d} \theta}-\operatorname{sgn}\left(X^{2}-U^{4}\right) \sqrt{2 \int_{\theta_{2}}^{\theta_{m}} \lambda \rho \mathrm{d} \theta}
$$

for the maximum temperature $\theta_{m}$.

\subsection{Determination of the spatial distribution of temperature and potential}

Following Greenwood and Williamson (1958), we define the function $\psi$ such that $\boldsymbol{J}=\boldsymbol{\nabla} \phi$. It follows that $\rho \nabla \psi=\nabla \phi$ and $\nabla^{2} \psi \equiv \operatorname{div} \boldsymbol{J}=0$. 
Squaring both sides of equation (4) and then differentiating with respect to $\theta$, we obtain

$$
\phi \frac{\mathrm{d} \phi}{\mathrm{d} \theta}=-\lambda \rho .
$$

We can then write

$$
\nabla \psi=\frac{1}{\rho} \nabla \phi=\frac{1}{\rho} \frac{\mathrm{d} \phi}{\mathrm{d} \theta} \nabla \theta=-\frac{\lambda}{\phi} \nabla \theta
$$

and hence

$$
\lambda \nabla \theta=-\phi \nabla \psi
$$

or

$$
\boldsymbol{q}=\phi \boldsymbol{J}
$$

We can also integrate equation (13) along a flux line, using equation (4) to eliminate $\phi$, with the result

$$
\psi=\operatorname{sgn}(\phi) \int_{\theta}^{\theta_{m}}\left(2 \int_{\theta}^{\theta_{m}} \lambda \rho \mathrm{d} \theta\right)^{-\frac{1}{2}} \lambda \mathrm{d} \theta .
$$

Notice that with this definition, the zero of $\psi$ is set to correspond with the isotherm $\theta=\theta_{m}$ and the sign of $\psi$ is set to be the same as that of $\phi$, which is determined by the procedure of the section 2.1. In particular, the values of $\psi$ on $S_{1}, S_{2}$ are

$$
\begin{gathered}
\psi_{1}=\operatorname{sgn}(U) \int_{\theta_{1}}^{\theta_{m}}\left(2 \int_{\theta}^{\theta_{m}} \lambda \rho \mathrm{d} \theta\right)^{-\frac{1}{2}} \lambda \mathrm{d} \theta \\
\psi_{2}=\operatorname{sgn}(U) \operatorname{sgn}\left(X^{2}-U^{4}\right) \int_{\theta_{2}}^{\theta_{m}}\left(2 \int_{\theta}^{\theta_{m}} \lambda \rho \mathrm{d} \theta\right)^{-\frac{1}{2}} \lambda \mathrm{d} \theta
\end{gathered}
$$

respectively, from equations (9) and (10).

These results reduce the problem to the determination of a harmonic function $\psi$ to satisfy the boundary conditions (17) and (18) on $S_{1}, S_{2}$ and the insulation condition $\partial \psi / d n=0$ on the remaining surfaces of $\Omega$. Once $\psi(\boldsymbol{r})$ is known as a function of position $r$, the corresponding temperature $\theta$ can be recovered by inverting equation (16).

The boundary value problem for $\psi$ is linear and it is convenient to make this explicit by defining

$$
\psi(\boldsymbol{r})=\frac{\psi_{1}+\psi_{2}}{2}+\frac{\psi_{2}-\psi_{1}}{2} g(\boldsymbol{r})
$$

where $g(\boldsymbol{r})$ is of the corresponding boundary value problem with $\nabla^{2} g=0$ and $g_{1}=-1, g_{2}=1$.

This terminology has the effect of decomposing the problem into two totally independent problems. The function $g(\boldsymbol{r})$ depends only upon the geometry of the problem and is independent of the material and the inhomogeneous boundary values $\theta_{1}, \theta_{2}, U$, whereas the relation between $\theta, \phi$ and $\psi$ depends upon the material properties and $\theta_{1}, \theta_{2}, U$, but it is independent of the geometry of the problem.

This decomposition has some important consequences. For example,

(i) All conductors made of the same material and subjected to the same boundary values $\theta_{1}, \theta_{2}, U$ will experience the same maximum temperature $\theta_{m}$ regardless of the shape and size of the conductor.
The maximum temperature in the body is uniquely determined by the procedure of the section 2.1, which makes no reference to the specific geometry of the body. In particular, the maximum temperature will be $\theta_{2}$ if $U^{4}>X^{2}$ (recall that we have labelled the surfaces so that $\theta_{2}>\theta_{1}$ ) and will be $\theta_{m}$ as determined by equation (11) if $U^{4}<$ $X^{2}$.

(ii) If two conductors $C, D$ of a given material transmit total currents $I^{C}, I^{D}$ for particular values of $\theta_{1}, \theta_{2}, U$, the ratio $I^{C} / I^{D}$ will be the same for all values of these quantities.

To prove this, we first note that the current density

$$
\boldsymbol{J}=\nabla \psi=\frac{\left(\psi_{2}-\psi_{1}\right)}{2} \nabla g
$$

from equation (19). The total current transmitted can therefore be written

$$
I=\int_{S_{1}} J_{n} \mathrm{~d} S_{1}=\left(\psi_{2}-\psi_{1}\right) I_{g}
$$

from equation (19), where the dimensionless current factor

$$
I_{g}=\frac{1}{2} \int_{S_{1}} \frac{\partial g}{\partial n} \mathrm{~d} S_{1}
$$

is the current that would flow through a conductor of the same shape with a unit potential difference between the surfaces $S_{1}, S_{2}$ and a temperature-independent electrical resistivity of unity.

It follows immediately that for given values of $\theta_{1}, \theta_{2}, U$, and hence of $\psi_{1}, \psi_{2}, I^{C} / I^{D}=I_{g}^{C} / I_{g}^{D}$ and this is the same for all values of $\psi_{1}, \psi_{2}$.

(iii) The temperature field $\theta$ in a body with boundary values $\theta_{1}, \theta_{2}, U$ can be mapped into a body of different geometry but the same boundary values using conformal mapping.

This follows because the only dependence on the geometry of the body is introduced through the function $g(\boldsymbol{r})$ and this function is harmonic with unit or zero gradient boundary values. These properties are presented under conformal transformation.

A major advantage of this result is that we can discuss the nonlinear aspects of the problem in the context of a simple one-dimensional geometry consisting of one or more bars with unidirectional conduction. This is particularly useful for the complex case where two or more conductors of different materials are involved.

\section{Conduction through composite bodies}

We now turn our attention to the case in which the conductor consists of two or more connected components of dissimilar materials, as shown in figure 1. The equipotential surface $S_{A}$ in $\Omega_{A}$ is maintained at temperature $\theta_{A}$ and $S_{B}$ in $\Omega_{B}$ is maintained at $\theta_{B}$. The two components are in intimate thermal and electrical contact at the interface $S_{I}$. All other surfaces of the bodies are assumed to 


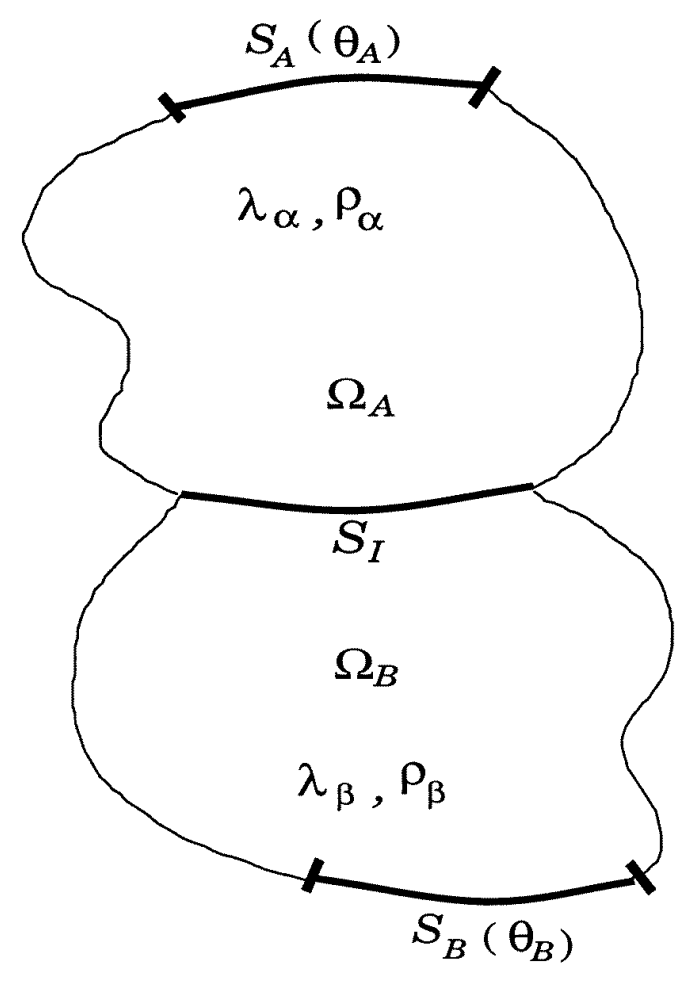

Figure 1. Configuration of dissimilar conductors in contact.

be insulated. Components $\Omega_{A}, \Omega_{B}$ are of materials $\alpha, \beta$ respectively, which have thermal conductivities $\lambda_{\alpha}, \lambda_{\beta}$ and electrical resistivities $\rho_{\alpha}, \rho_{\beta}$, all of which are functions of temperature. A potential difference $U$ is applied between $S_{A}$ and $S_{B}$ and we wish to determine the current that will flow and the resulting temperature and potential fields.

The analysis of section 2 can be applied separately to $\Omega_{A}, \Omega_{B}$, if and only if the interface $S_{I}$ is an equipotential, isothermal surface. This in turn will be the case as long as $S_{I}$ is a surface of constant $g=g_{I}$ in the domain $\Omega_{A} \cup \Omega_{B}$ with $g_{A}=-1, g_{B}=1$. This places important restrictions on the applicability of the method developed in this section and therefore deserves some preliminary discussion. An important general class satisfying the condition is that in which the interface is a plane of symmetry between two geometrically identical conductors. The special case where the conductors are half spaces in contact at a number of regions in their common plane is discussed in more detail in section 4 below, but the method is equally applicable to finite conductors, provided they are geometrically symmetrical. Numerous other special cases can be identified by appealing to classical solutions of Laplace's equation in two and three dimensions and locating the interface on a known isopotential surface. Such cases include, for example, two curved bars of constant radius and similar but arbitrary cross section contacting on a common transverse plane, or the contact of two annular wedge-shaped regions contacting on a common circular boundary.

\subsection{Conditions at the interface}

If $S_{I}$ is the equipotential surface $g=g_{I}$ in $\Omega_{A} \cup \Omega_{B}$, we can construct the functions $g_{A}, g_{B}$ for the domains $\Omega_{A}, \Omega_{B}$ respectively through the linear mapping

$$
g_{A}=\frac{2 g+\left(1-g_{I}\right)}{1+g_{I}}, g_{B}=\frac{2 g-\left(1+g_{I}\right)}{1-g_{I}} .
$$

The current density is required to be continuous throughout the interface $S_{I}$ and hence

$$
\left(\frac{\partial \psi^{\alpha}}{\partial n}\right)_{I}=\left(\frac{\partial \psi^{\beta}}{\partial n}\right)_{I}
$$

where the superscripts $\alpha, \beta$ refer to the potentials in materials $\alpha, \beta$ respectively. Using equation (19) and (23), this condition reduces to

$$
\frac{\psi_{I}^{\alpha}-\psi_{A}^{\alpha}}{1+g_{I}}\left(\frac{\partial g}{\partial n}\right)_{I}=\frac{\psi_{B}^{\beta}-\psi_{I}^{\beta}}{1-g_{I}}\left(\frac{\partial g}{\partial n}\right)_{I}
$$

which will be satisfied throughout $S_{I}$ as long as

$$
\frac{\psi_{I}^{\alpha}-\psi_{A}^{\alpha}}{1+g_{I}}=\frac{\psi_{B}^{\beta}-\psi_{I}^{\beta}}{1-g_{I}}
$$

This relation can also be expressed in terms of the current factor $I_{g}$ of equation (22) in the form

$$
I=\left(\psi_{I}^{\alpha}-\psi_{A}^{\alpha}\right) I_{g}^{A}=\left(\psi_{B}^{\beta}-\psi_{I}^{\beta}\right) I_{g}^{B}
$$

where $I$ is the total current flowing through the composite conductor.

We also require continuity of heat flux at the interface and hence

$$
\lambda_{\alpha}\left(\theta_{I}\right)\left(\frac{\partial \theta^{\alpha}}{\partial n}\right)_{I}=\lambda_{\beta}\left(\theta_{I}\right)\left(\frac{\partial \theta^{\beta}}{\partial n}\right)_{I}
$$

where $\theta_{I}$ is the temperature on $S_{I}$.

Using the result (14), this condition can be written

$$
\phi_{I}^{\alpha}\left(\frac{\partial \psi^{\alpha}}{\partial n}\right)_{I}=\phi_{I}^{\beta}\left(\frac{\partial \psi^{\beta}}{\partial n}\right)_{I}
$$

and hence, using equation (24)

$$
\phi_{I}^{\alpha}=\phi_{I}^{\beta}
$$

It should be emphasized that the separate potentials $\phi^{\alpha}, \phi^{\beta}$ have been conventionally chosen such that they are zero at the points (inside or outside the body) where $\theta=\theta_{m}^{\alpha}$, $\theta_{m}^{\beta}$ respectively. Thus, there is no independent physical reason to expect continuity of $\phi$ at the interface. However, the above argument shows that this continuity is required as a consequence of the continuity of heat flux and current density. The condition is necessary and sufficient in the sense that any two of equations (24), (28) and (30) imply the third. 


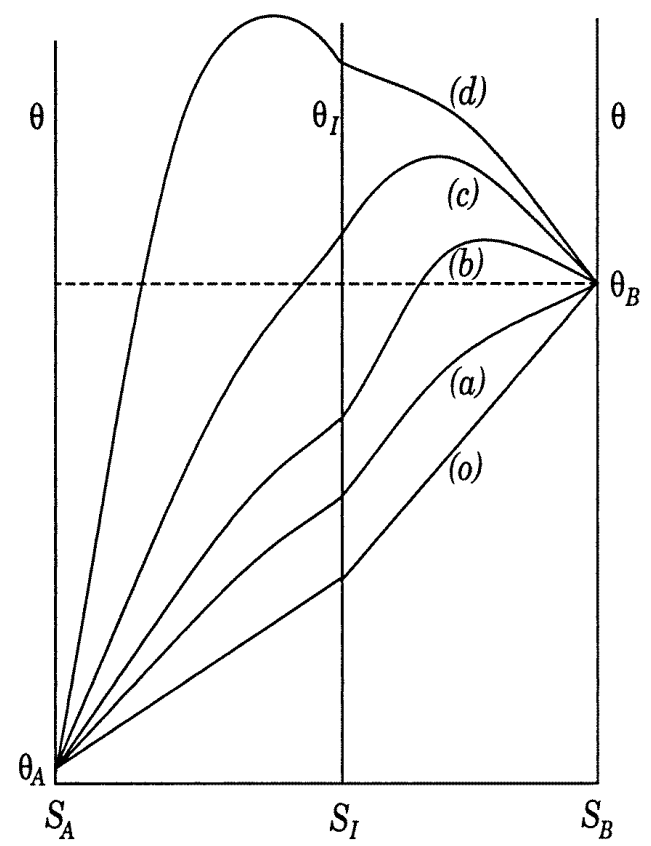

Figure 2. Forms of temperature field that can evolve with increasing potential difference when $\theta_{B}>\theta_{A}$.

\subsection{Location of the maximum temperature}

If we label the components such that $\theta_{B}>\theta_{A}$, there are four possible scenarios, illustrated schematically for the onedimensional problem in figure 2 (curves $(a)-(d)$ ). These are defined by the conditions:

(a) The temperature increases monotonically through both components and $\theta_{A}<\theta_{I}<\theta_{B}$.

(b) The temperature increases monotonically through $\Omega_{A}$, the maximum temperature $\theta_{m}^{\beta}$ occurs in $\Omega_{B}$ and $\theta_{A}<$ $\theta_{I}<\theta_{B}$.

(c) As in case (b), the temperature increases monotonically through $\Omega_{A}$ and the maximum temperature $\theta_{m}^{\beta}$ occurs in $\Omega_{B}$, but $\theta_{A}<\theta_{B}<\theta_{I}$.

(d) The maximum temperature $\theta_{m}^{\alpha}$ occurs in $\Omega_{A}$, the temperature falls monotonically through $\Omega_{B}$ and $\theta_{A}<\theta_{B}<$ $\theta_{I}$.

There are also three special transitional states between cases. For example, between $(c)$ and $(d)$ there is the special case $\theta_{I}=\theta_{m}^{\alpha}=\theta_{m}^{\beta}=\theta_{m}^{\beta}>\theta_{B}>\theta_{A}$.

It is easily verified that all other scenarios lead to a violation of the continuity condition (28). For example, if $\theta_{m}^{\alpha}$ occurred in $\Omega_{A}$ and $\theta_{m}^{\beta}$ occurred in $\Omega_{B}$, the gradient $\partial \theta / \partial n$ would be of different sign on the two sides of the interface.

There is a natural progression from case $(a) \rightarrow(b) \rightarrow$ $(c) \rightarrow(d)$ as the potential difference $|U|$ is increased. At very low potential differences, the Joule heating will be very small and the temperature will vary linearly through the one-dimensional conductors as shown in curve $(o)$. Increased current leads to increase in temperature everywhere and in particular to an increase in the interface temperature $\theta_{I}$. However, a local maximum must occur in $\Omega_{B}$ before $\theta_{I}$ reaches $\theta_{B}$ and a local maximum cannot occur in $\Omega_{A}$ until $\theta_{I}>\theta_{B}$.

\subsection{Solution for $\theta_{m}^{\alpha}, \theta_{m}^{\beta}$}

The most natural statement of the problem of figure 1 is to prescribe the geometry of the conductors, the boundary values $\theta_{A}, \theta_{B}$ and the potential difference $U$. However, in view of the sign differences that occur in certain quantities between the cases $(a)-(d)$ above, it is more convenient to regard $\theta_{A}, \theta_{B}$ and the interface temperature $\theta_{I}$ as independent variables and solve for the potential difference $|U|$ required to produce it. An increase in $|U|$ causes an increase in temperature at all points, including $\theta_{I}$, so the relation between $|U|$ and $\theta_{I}$ is monotonic and hence easily inverted numerically if required.

If $\theta_{A}, \theta_{B}, \theta_{I}$ are prescribed, two nonlinear equations for $\theta_{m}^{\alpha}, \theta_{m}^{\beta}$ can be written down from the continuity conditions (27) and (30), using equations (9) and (10) and equations (17) and (18). The appropriate signs to use in these definitions depend on which of cases $(a)-(d)$ is under consideration. For $\Omega_{A}, \theta_{I}>\theta_{A}$ for all cases; therefore we identify $\theta_{I}$ with $\theta_{A}$ and $\theta_{2}$ with $\theta_{I}$. The maximum temperature occurs in $\Omega_{A}$ only for case $(d)$ and hence for this case the term $\operatorname{sgn}\left(X^{2}-U^{4}\right)=-1$ in equations (10) and (18), whereas for cases $(a)-(c)$ it is +1 . We therefore conclude that

$$
\begin{gathered}
\phi_{A}^{\alpha}=\operatorname{sgn}(U) \sqrt{2 \int_{\theta_{A}}^{\theta_{m}^{\alpha}} \lambda_{\alpha} \rho_{\alpha} \mathrm{d} \theta} \\
\phi_{I}^{\alpha}=\operatorname{sgn}(U) j_{1} \sqrt{2 \int_{\theta_{I}}^{\theta_{m}^{\alpha}} \lambda_{\alpha} \rho_{\alpha} \mathrm{d} \theta} \\
\psi_{A}^{\alpha}=\operatorname{sgn}(U) \int_{\theta_{A}}^{\theta_{m}^{\alpha}}\left(2 \int_{\theta}^{\theta_{m}^{\alpha}} \lambda_{\alpha} \rho_{\alpha} \mathrm{d} \theta\right)^{-\frac{1}{2}} \lambda_{\alpha} \mathrm{d} \theta \\
\psi_{I}^{\alpha}=\operatorname{sgn}(U) j_{1} \int_{\theta_{I}}^{\theta_{m}^{\alpha}}\left(2 \int_{\theta}^{\theta_{m}^{\alpha}} \lambda_{\alpha} \rho_{\alpha} \mathrm{d} \theta\right)^{-\frac{1}{2}} \lambda_{\alpha} \mathrm{d} \theta
\end{gathered}
$$

where $j_{1}=+1$ for cases $(a)-(c)$ and -1 for case $(d)$.

For $\Omega_{B}, \theta_{B}>\theta_{I}$ for cases $(a)$ and $(b)$, whereas $\theta_{B}<\theta_{I}$ for cases $(c)$ and $(d)$. In the latter case, we therefore have to identify $\theta_{1}$ with $\theta_{B}$ and $\theta_{2}$ with $\theta_{I}$, with the result that $\operatorname{sgn}\left(U_{B}\right)=-\operatorname{sgn}(U)$, since $U_{B}=\phi_{1}-\phi_{2}=\phi_{B}-\phi_{I}$ in cases $(c)$ and $(d)$. Using this result and proceeding as in the case of $\Omega_{A}$ above, we conclude that

$$
\begin{gathered}
\phi_{B}^{\beta}=\operatorname{sgn}(U) j_{2} \sqrt{2 \int_{\theta_{B}}^{\theta_{m}^{\beta}} \lambda_{\beta} \rho_{\beta} \mathrm{d} \theta} \\
\phi_{I}^{\beta}=\operatorname{sgn}(U) j_{1} \sqrt{2 \int_{\theta_{I}}^{\theta_{m}^{\beta}} \lambda_{\beta} \rho_{\beta} \mathrm{d} \theta} \\
\psi_{B}^{\beta}=\operatorname{sgn}(U) j_{2} \int_{\theta_{B}}^{\theta_{m}^{\beta}}\left(2 \int_{\theta}^{\theta_{m}^{\beta}} \lambda_{\beta} \rho_{\beta} \mathrm{d} \theta\right)^{-\frac{1}{2}} \lambda_{\beta} \mathrm{d} \theta \\
\psi_{I}^{\beta}=\operatorname{sgn}(U) j_{1} \int_{\theta_{I}}^{\theta_{m}^{\beta}}\left(2 \int_{\theta}^{\theta_{m}^{\beta}} \lambda_{\beta} \rho_{\beta} \mathrm{d} \theta\right)^{-\frac{1}{2}} \lambda_{\beta} \mathrm{d} \theta
\end{gathered}
$$

where $j_{2}=+1$ for case $(a)$ and -1 for cases $(b)-(d)$. 
Table 1.

\begin{tabular}{lllll}
\hline & $(a)$ & $(b)$ & $(c)$ & $(d)$ \\
\hline$j_{1}=$ & +1 & +1 & +1 & -1 \\
$j_{2}=$ & +1 & -1 & -1 & -1 \\
$j_{3}=$ & +1 & -1 & -1 & +1 \\
\hline
\end{tabular}

Substituting these results into equation (30), we find that for all four cases

$$
\sqrt{2 \int_{\theta_{I}}^{\theta_{m}^{\alpha}} \lambda_{\alpha} \rho_{\alpha} \mathrm{d} \theta}=\sqrt{2 \int_{\theta_{I}}^{\theta_{m}^{\beta}} \lambda_{\beta} \rho_{\beta} \mathrm{d} \theta}
$$

and hence

$$
\int_{\theta_{I}}^{\theta_{m}^{\alpha}} \lambda_{\alpha} \rho_{\alpha} \mathrm{d} \theta=\int_{\theta_{I}}^{\theta_{m}^{\beta}} \lambda_{\beta} \rho_{\beta} \mathrm{d} \theta
$$

Corresponding results for equation (27) can be written in the form

$$
\begin{aligned}
I_{g}^{A}[ & \int_{\theta_{I}}^{\theta_{m}^{\alpha}}\left(2 \int_{\theta}^{\theta_{m}^{\alpha}} \lambda_{\alpha} \rho_{\alpha} \mathrm{d} \theta\right)^{-\frac{1}{2}} \lambda_{\alpha} \mathrm{d} \theta \\
& \left.-j_{1} \int_{\theta_{A}}^{\theta_{m}^{\alpha}}\left(2 \int_{\theta}^{\theta_{m}^{\alpha}} \lambda_{\alpha} \rho_{\alpha} \mathrm{d} \theta\right)^{-\frac{1}{2}} \lambda_{\alpha} \mathrm{d} \theta\right] \\
= & I_{g}^{B}\left[j_{3} \int_{\theta_{B}}^{\theta_{m}^{\beta}}\left(2 \int_{\theta}^{\theta_{m}^{\beta}} \lambda_{\beta} \rho_{\beta} \mathrm{d} \theta\right)^{-\frac{1}{2}} \lambda_{\beta} \mathrm{d} \theta\right. \\
& \left.-\int_{\theta_{I}}^{\theta_{m}^{\beta}}\left(2 \int_{\theta}^{\theta_{m}^{\beta}} \lambda_{\beta} \rho_{\beta} \mathrm{d} \theta\right)^{-\frac{1}{2}} \lambda_{\beta} \mathrm{d} \theta\right]
\end{aligned}
$$

where $j_{3}=+1$ for cases $(a)$ and $(d)$ and -1 for cases $(b)$ and $(c)$. The values of $j_{1}, j_{2}$ and $j_{3}$ are summarized in table 1 .

Before solving equations (40) and (41) for $\theta_{m}^{\alpha}, \theta_{m}^{\beta}$, it is convenient to determine the range of values of $\theta_{I}$ for each of the four cases $(a)-(d)$. The transition between cases $(a)$ and $(b)$ is obtained by substituting $\theta_{m}^{\beta}=\theta_{B}$ into equations (40) and (41) and solving for $\theta_{m}^{\alpha}, \theta_{I}$, whilst that between cases $(b)$ and $(c)$ corresponds to $\theta_{I}=\theta_{B}$. The transition between cases $(c)$ and $(d)$ occurs when $\theta_{I}=\theta_{m}^{\alpha}=\theta_{m}^{\beta}$. With these values, equation (40) is satisfied identically and equation (41) yields the equation

$$
R \equiv \frac{\int_{\theta_{B}}^{\theta_{I}}\left(2 \int_{\theta}^{\theta_{I}} \lambda_{\beta} \rho_{\beta} \mathrm{d} \theta\right)^{-\frac{1}{2}} \lambda_{\beta} \mathrm{d} \theta}{\int_{\theta_{A}}^{\theta_{I}}\left(2 \int_{\theta}^{\theta_{I}} \lambda_{\alpha} \rho_{\alpha} \mathrm{d} \theta\right)^{-\frac{1}{2}} \lambda_{\alpha} \mathrm{d} \theta}=\frac{I_{g}^{A}}{I_{g}^{B}}
$$

for the critical value of $\theta_{I}$ at the transition. Notice that for any given materials and values of $\theta_{A}, \theta_{B}, R$ will have a maximum value $R_{m}$ in the domain $\theta_{B}<\theta_{I}<\infty$. It follows that there will be no transition to case $(d)$ if $I_{g}^{A} / I_{g}^{B}>R_{m}$. Instead, case $(c)$ will persist for all values $\theta_{I}>\theta_{B}$. This argument also shows that case $(d)$ is more likely to occur if the ratio $I_{g}^{A} / I_{g}^{B}$ is small. This is intuitively reasonable, since in the limit where $I_{g}^{B} \rightarrow \infty$. We must recover the case of a single conductor of material $A$, in which the maximum temperature will occur sufficiently high potential $U$.

Once $\theta_{m}^{\alpha}, \theta_{m}^{\beta}$ have been found, the total potential $U$ can then be recovered from the relation $U=\phi_{B}^{\beta}-\phi_{A}^{\alpha}$, which with equations (31) and (35) gives

$$
|U|=\sqrt{2 \int_{\theta_{A}}^{\theta_{m}^{\alpha}} \lambda_{\alpha} \rho_{\alpha} \mathrm{d} \theta}-j_{2} \sqrt{2 \int_{\theta_{B}}^{\theta_{m}^{\beta}} \lambda_{\beta} \rho_{\beta} \mathrm{d} \theta} .
$$

\subsection{A one-dimensional example}

To illustrate the use of the method, we consider the onedimensional example of two cylinders of unit length and unit cross-sectional area occupying the regions $-1<x<$ $0,0<x<1$ respectively. The cylinders make contact at the common interface $x=0$ and the surfaces $S_{A}, S_{B}$ correspond to $x=-1,1$ respectively. With this geometry, we have $g(x)=x$ and the current factor $I_{g}=\frac{1}{2}$.

We first consider the case where the cylinder $\Omega_{A}$ is aluminium $\left(\lambda=240 \mathrm{~W} \mathrm{~m}^{-1} \mathrm{~K}^{-1}, \rho=2.5 \times 10^{-8}(1+\right.$ $0.004 \theta) \Omega \mathrm{m})$ and $\Omega_{B}$ is brass $\left(\lambda=119 \mathrm{~W} \mathrm{~m}^{-1} \mathrm{~K}^{-1}\right.$, $\left.\rho=5.9 \times 10^{-8}(1+0.00346 \theta) \Omega \mathrm{m}\right)$.

Figure $3(a)$ shows the temperature distribution in the cylinders for $\theta_{A}=0, \theta_{B}=100$ and various values of $\theta_{I}$ or $|U|$. The corresponding relation between $|U|$ and $\theta_{I}$ is shown in figure $3(b)$, which also shows the maximum temperature $\theta_{m}$. As anticipated, $\theta_{I}$ increases monotonically with $|U|$ and there is a natural progression from case $(a)$ to $(b)$ to $(c)$. However, for this configuration, case $(d)$ is never achieved because $R_{m}=0.493<1$ and $I_{g}^{A}=I_{g}^{B}$.

At large $\theta_{I}, R$ is relatively insensitive to the boundary temperatures $\theta_{A}, \theta_{B}$ and we generally have $R_{m}<1$ for $\lambda_{\beta} \rho_{\beta}<\lambda_{\alpha} \rho_{\alpha}$ and $R_{m}>1$ for $\lambda_{\beta} \rho_{\beta}<\lambda_{\alpha} \rho_{\alpha}$. Thus, by interchanging the materials but retaining the values of $\theta_{A}, \theta_{B}$, we obtain a system that exhibits the full sequence of cases $(a),(b),(c),(d)$, as shown in figures $4(a)$ and $4(b)$.

Timisit (1988) gives an approximate solution of the two-material problem, based on the assumption that the maximum temperature will always coincide with the midpotential point $|U| / 2$. The present exact method was used to assess the accuracy of this approximation in a variety of examples. When the conductors are of equal size and have equal boundary temperatures, Timisit's approximation predicts the maximum temperature with an accuracy that is generally better than $6 \%$. However, the error increases when there is a significant difference between $I_{g}^{A}$ and $I_{g}^{B}$ or between $\theta_{A}$ and $\theta_{B}$. For example, with $\theta_{A}=0{ }^{\circ} \mathrm{C}, \theta_{B}=$ $100{ }^{\circ} \mathrm{C}$ and $I_{g}^{B}=I_{g}^{A}$, his method gives errors of up to $25 \%$.

\section{Constriction resistance}

We now examine the consequences of these results for the problem of conduction of electricity between two large conductors across the constriction resistance associated with the roughness of the contacting surfaces. For this purpose, we assume that the conductors can be represented by halfspaces, with contact occurring at one or more circular 'actual contact areas' at the interface.

The conductors will generally be assumed to be of different materials and/or to have different temperatures at infinity, but the geometry of the system is symmetrical about the interfacial plane and it follows that the conditions imposed in section 3.1 are satisfied. In fact, the interface will be the surface $g=g_{I}=0$ in the full geometry, leading to $g_{A}=2 g+1$ and $g_{B}=2 g-1$ in equation (23). The symmetry also guarantees that

$$
I_{g}^{A}=I_{g}^{B}=2 I_{g}
$$




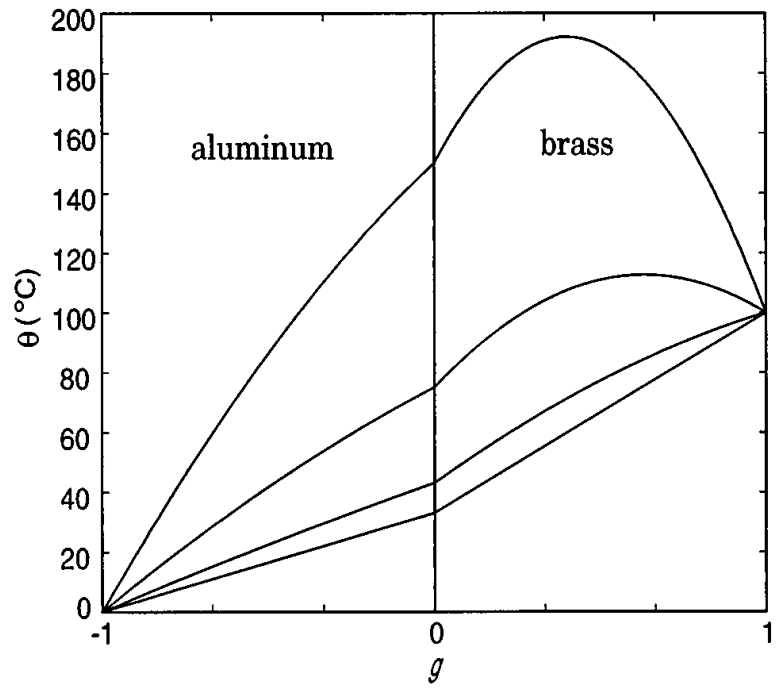

(a)

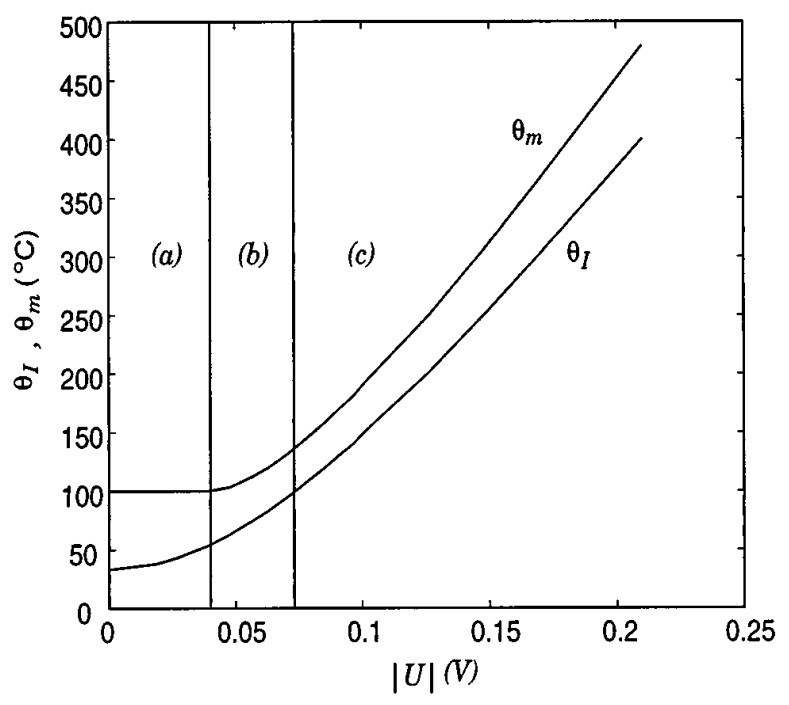

(b)

Figure 3. Electrical conduction through equal contacting cylinders of aluminium and brass: (a) temperature field;

(b) relation between $|U|, \theta_{l}$ and $\theta_{m}$.

It follows that the actual contact areas constitute a single isothermal, equipotential surface for all actual contact configurations, and hence that the temperature and potential fields can be mapped into the corresponding onedimensional solution for two conductors of equal length.

\subsection{Single contact area}

For the case of a single circular contact of radius $a$, the function $g$ can be expressed in ellipsoidal coordinates $\xi, \eta$ as

$$
g=-\frac{2}{\pi} \tan ^{-1} \xi
$$

(Greenwood and Williamson 1958) where $\xi, \eta$ are related to the cylindrical polar coordinates $r, z$ through

$$
r=a \sqrt{\left(1+\xi^{2}\right)\left(1-\eta^{2}\right)}, z=a \xi \eta .
$$

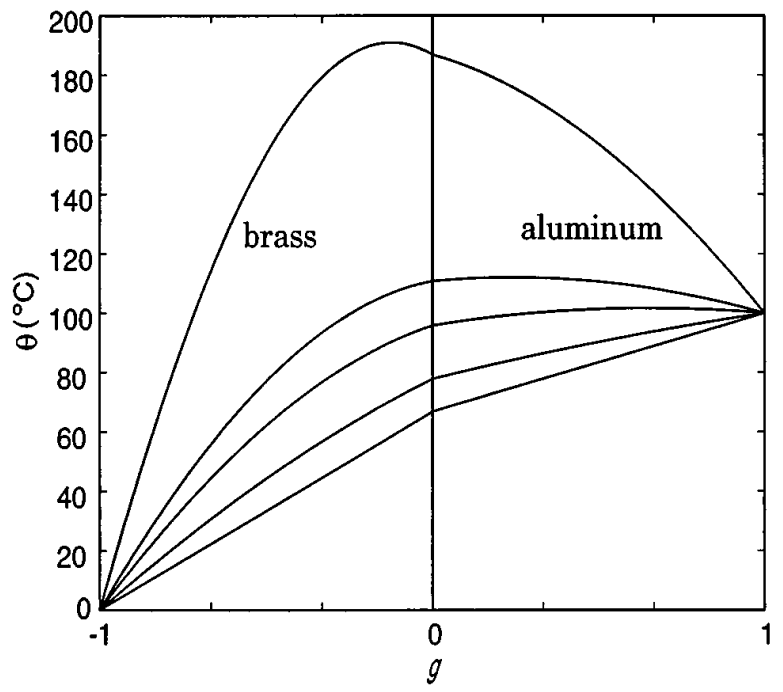

(a)

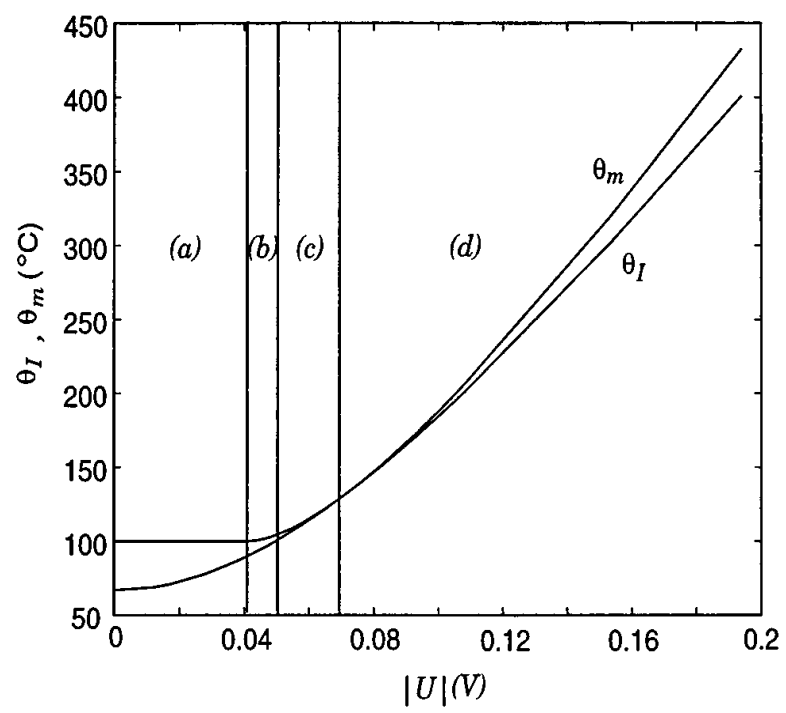

(b)

Figure 4. Electrical conduction through equal contacting cylinders of brass and aluminium: (a) temperature field; (b) relation between $|U|, \theta_{l}$ and $\theta_{m}$

Using these results, the temperatures and potentials of the example of the section 3.4 can be used to predict the corresponding fields for the contact of two half-spaces at a single circular area. Figure 5 shows the temperature distribution $(a)$ along the $z$-axis and $(b)$ in the plane $z=0$ for the two conductors, for the case where $\Omega_{A}$ is aluminium, $\Omega_{B}$ is brass and $\theta_{A}=0{ }^{\circ} \mathrm{C}, \theta_{B}=100{ }^{\circ} \mathrm{C}$ and $|U|=0.1 \mathrm{~V}$. The maximum temperature in this case occurs on an ellipsoidal surface in $\Omega_{B}$.

\subsection{Multiple contact areas}

In the contact of rough conforming surfaces, there will generally be a statistical distribution of actual contact areas of various shapes and sizes. Many authors (Greenwood and Williamson 1966, Cooper et al 1969, Onions and Archard 1973, Majumdar and Tien 1991) have discussed methods 


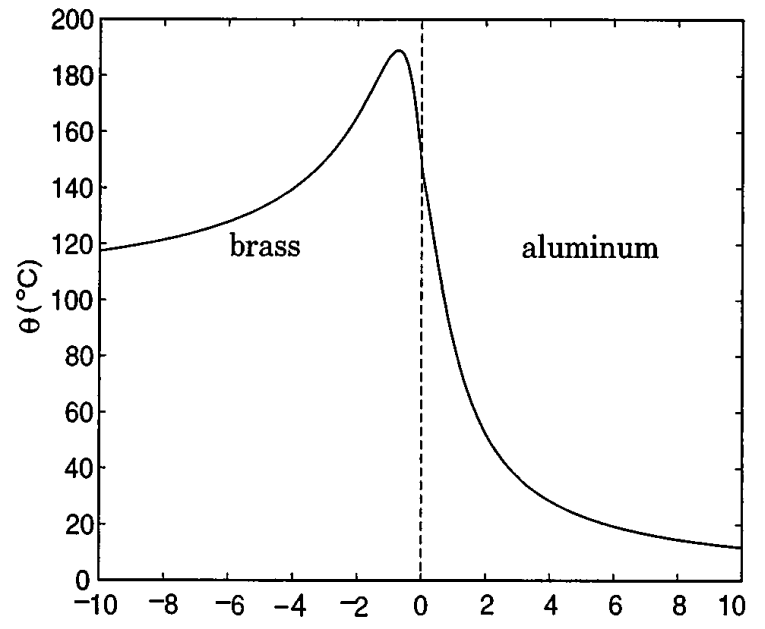

(a)

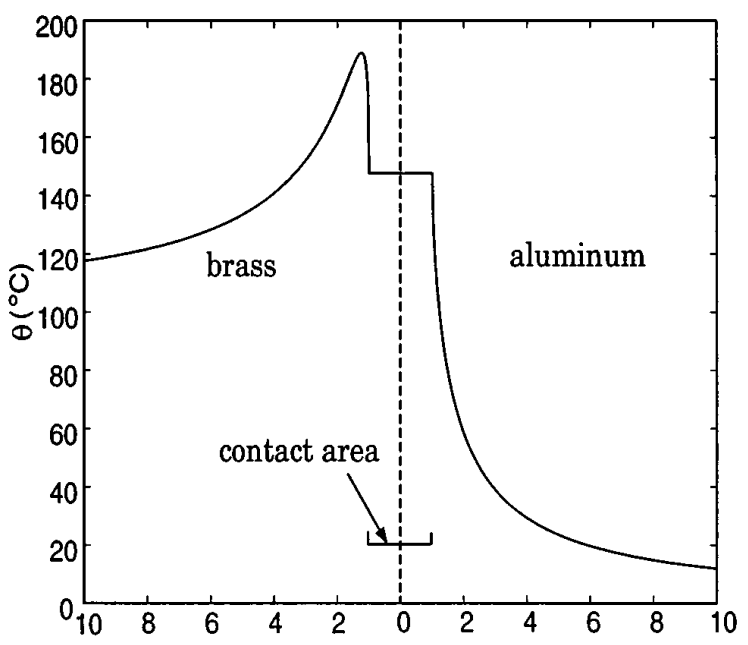

(b)

Figure 5. Temperature field within two dissimilar conductors making contact at a circular area: (a) along the axis of symmetry; (b) in the plane of the interface.

to determine the thermal and electrical contact resistance in such cases, using properties of the surfaces obtained from profilometry. These papers deal exclusively with the linear problem in which the material properties are independent of temperature.

One of the simplest approaches, introduced by Greenwood (1966) is to superpose the single contact area solution defined by equation (45), but use the point source solution to approximate the effect of current flow through any one contact area on the potential at the others. We proceed directly to a solution for the potential $g$ by requiring a potential difference of unity between the interface and the extremity of body 1 .

In this case, Greenwood's approximation gives the value of $g$ at the $i$ th contact area as

$$
g_{i}=-1+\sum_{j=1}^{N} A_{i j} I_{g j}
$$

where

$$
\begin{array}{rlrl}
A_{i j} & =\frac{1}{4 a_{i}} & & j=i \\
& =\frac{1}{2 \pi s_{i j}} & j \neq i
\end{array}
$$

$a_{i}$ is the radius of the $i$ th contact area and

$$
s_{i j}=\left|\boldsymbol{r}_{i}-\boldsymbol{r}_{j}\right|
$$

is the distance between the centres of the $i$ th and $j$ th contact areas.

Symmetry requires that $g_{i}=0$ for all $i$ and hence

$$
I_{g j}=\sum_{i=1}^{N} C_{j i}
$$

where the symmetric matrix $\boldsymbol{C}=\boldsymbol{A}^{-1}$. The total current factor can also be written

$$
I_{g}=\sum_{j=1}^{N} I_{g j}=\sum_{j=1}^{N} \sum_{i=1}^{N} C_{j i}
$$

Once $I_{g j}$ is known, the potential at a general point can be written by superposition. Suppose we take the origin of coordinates to be at the centre of the $i$ th contact area. Then the potential at points relatively near to the $i$ th contact area can be written

$$
g(\boldsymbol{r})=-1-\frac{I_{g i}}{4 a_{i}}\left(\frac{2}{\pi} \tan ^{-1} \xi-1\right)+\frac{1}{2 \pi} \sum_{j \neq i}^{N} \frac{I_{g j}}{\left|\boldsymbol{r}-\boldsymbol{r}_{j}\right|} .
$$

Since the origin can be chosen arbitrarily, such an expression can be written for all points in the domain.

These results completely define the function $g$ for the domain consisting of two half-spaces in contact at a set of sparsely distributed circular contact areas on the interfacial plane. As in the section 4.1, it is then a routine process to map the temperature fields from the corresponding linear problem of the section 3.4 into the new domain. The decoupling of the linear and nonlinear problems guarantees that the maximum temperature and the interfacial temperature for multispot contact will depend only on the boundary conditions $\theta_{A}, \theta_{B},|U|$ and will be independent of the size and spatial distribution of contact areas. The total current flow will depend upon these distributions through equations (27), (44) and (51).

\section{Conclusions}

We have demonstrated how the method of Greenwood and Williamson can be used to determine the steadystate temperature and potential fields in a system of two contacting conductors with temperature-dependent electrical resistivity and thermal conductivity. The 
nonlinear problem decouples into the solution of a pair of nonlinear algebraic equations involving the boundary values and the material properties, followed by a linear mapping of the resulting one-dimensional solution into the actual conductor geometry. Thus, the method can be applied to conductors of arbitrary geometry, provided that (i) the current enters and leaves the conductor through two equipotential isothermal surfaces, (ii) the remaining boundaries of the conductor are thermally and electrically insulating and (iii) the interface(s) between different materials would be equipotential surfaces in the corresponding linear problem. The method can in principle be extended to systems involving more than two conductors of dissimilar materials, subject to the same restrictions.

\section{Acknowledgments}

The authors are pleased to acknowledge the support of the Advanced Technology Program through the 'Intelligent Resistance Welding' Consortium.

\section{References}

Bryant M D 1994 IEEE Trans. Comp. Hybrids Manuf. Technol. 17 86-95

Cooper M G, Mikic B B and Yovanovich M M 1969 Int. J. Heat Mass Transfer 12 279-300

Diesselhorst H 1900 Ann. Phys., Lpz. 1 312-25

Fournet G 1997 J. Physique III 7 2003-29

Greenwood J A 1966 Br. J. Appl. Phys. 17 1621-32

Greenwood J A and Williamson J B P 1958 Proc. R. Soc. A 246 13-31

-1966 Proc. R. Soc. A 345 300-19

Holm R 1967 Electrical Contacts (New York: Springer)

Kohlrausch F 1990 Ann. Phys., Lpz. 1 132-58

Majumdar A and Tien C L 1991 ASME J. Tribol. 113 516-25

Onions R A and Archard J F 1973 J. Phys. D: Appl. Phys. 6 289-304

Runde M 1987 IEEE Trans. Hybrids Manuf. Technol. 10 89-99

Thornton P H, Krause A R and Davies R G 1996 Welding J. 75 402s-12s

Timisit R S 1988 IEEE Trans. Comp. Hybrids Manuf. Technol. 11 43-53

Yune Y G and Bryant M D 1988 IEEE Trans. Comp. Hybrids Manuf. Technol. 11 91-100 